\title{
Location-Based Resource Allocation for OFDMA Cognitive Radio Systems
}

\author{
Haewoon Nam \\ Mobile Devices Technology Office \\ Motorola Inc. \\ Austin, TX, USA \\ Email: haewoon_nam@ieee.org
}

\author{
Mahdi Ben Ghorbel and Mohamed-Slim Alouini \\ Electrical Engineering Program \\ King Abdullah University of Sciences and Technology \\ Thuwal, Makkah Province, Saudi Arabia \\ Email: \{mahdi.benghorbel,slim.alouini\}@kaust.edu.sa
}

\begin{abstract}
In cognitive radio systems, in order for the secondary users to opportunistically share the spectrum without interfering the primary users, an accurate spectrum measurement and a precise estimation of the interference at the primary users are necessary but are challenging tasks. Since it is impractical in cognitive radio systems to assume that the channel state information of the interference link is available at the cognitive transmitter, the interference at the primary users is hard to be estimated accurately. This paper introduces a resource allocation algorithm for OFDMA-based cognitive radio systems, which utilizes location information of the primary and secondary users instead of the channel state information of the interference link. Simulation results show that it is indeed effective to incorporate location information into resource allocation so that a nearoptimal capacity is achieved.
\end{abstract}

Index Terms - Cognitive radios and resource allocation

\section{INTRODUCTION}

Cognitive radio systems have received a great deal of attentions recently as future radios to bring a flexible operating intelligence to the devices such that they can coexist with the existing systems with no interference and share the spectrum [1], [2]. It requires them a capability to find a spectrum hole and a scheduling flexibility to avoid the occupied spectrum and selectively use the empty spectrum. Since orthogonal frequency division multiple access (OFDMA) is widely used in various wireless multiuser systems due to high spectral efficiency and such a scheduling flexibility, OFDMA systems are attractive for cognitive radio systems.

Location information is very useful in cognitive radio systems in various ways [3]. First of all, location information allows the secondary users to compute the relative distance between the primary and the secondary users, which greatly helps the secondary users 1) estimate pathloss exponent with the help of receive power measurement, 2) perform a more precise measurement of the spectrum occupancy, 3) determine the minimum transmit power level for a reliable link between the secondary users, 4) determine angle of arrival/departure toward primary users and use beamforming techniques to reduce the interference to the primary users if multiple antennas are available, and 5) optimize the cognitive radio networks in order to maximize the spectrum usage and the spatial reuse.

This work is funded by King Abdullah University of Sciences and Technology.
A variety of localization techniques are introduced in the literature (see [4]-[6] and the references therein). Location information about the primary users can be delivered to the secondary base station by the primary base station if available using a backhaul link or estimated by a cooperation of multiple secondary users based on a receive power measurement and a triangulation technique. Location of each secondary user may be determined autonomously using global positioning system (GPS) or estimated based on a power measurement of pilot signals from the surrounding beacons. In this paper, we assume that location information of the primary and the secondary users is available to the secondary base stations where a resource allocation algorithm is executed.

Among the aformetioned benefits of location information, this paper focuses on how the relative location between the primary users and the secondary transmitter can be exploited for resource allocation in cognitive radio networks under log-normal shadowing. Additionally, the impacts of different user distributions in a cellular environment and a correlation between subchannels on the performance are considered.

An extensive research has been recently performed for resource allocation in cognitive radio networks [7]-[9]. Many of them have assumed that the channel gains of the interference links between secondary transmitters and primary users are available. In order to estimate the interference at primary users accurately, a secondary transmitter needs to know the channel state information (CSI) of the interference link. However, since it is impractical in cognitive radio systems to assume that the primary users send feedbacks to the secondary transmitter, an accurate estimate of the CSI of the interference link may not be possible in practice. Thus, a resource allocation algorithm for the secondary users that does not depend on the CSI of the interference link is of paramount importance. Without any knowledge about the interference link, the secondary transmitter may need to avoid transmission over the frequency bands used by the primary users (overlay). But the secondary transmitter may use those frequency bands when the interference constraint is characterized by the location information.

\section{System And Channel Model}

Consider cellular cognitive radio networks that consist of a secondary base station (single cell environment) and $K$ 
secondary users who aim to opportunistically use the spectrum occupied by the primary users without causing an interference to the primary users. Assume primary users are in time division duplex (TDD) mode, where the same frequency band is used for transmission and reception, and the cognitive networks use OFDMA with $L$ subchannels and a wider system bandwidth than that of the primary users due to the interference mitigation based on frequency diversity and the capability of a selective use of unoccupied subchannels by the primary users, where subchannel is defined as a group of subcarriers. In [10], interference temperature is defined as the radio frequency (RF) power measured at a receiving antenna per unit bandwidth and indicates the tolerable interference level at the primary user's receiver.

We assume omni-directional transmission for primary users as well as secondary users. For simplicity, we also assume that there is a single primary user, but the analysis shown in this paper can be easily extended to the case of multiple primary users each with a different interference temperature level.

Consider 2 dimensional coordinate $\{x, y\}$ where primary and secondary users are placed in the same horizontal plane (no height elevation). Let $\left\{x_{1}^{(p)}, y_{1}^{(p)}\right\}$ denote the location of the single primary user, $\left\{x_{k}^{(c)}, y_{k}^{(c)}\right\}$ the location of the secondary user $k$, and $\left\{x_{0}^{(c)}, y_{0}^{(c)}\right\}$ the location of the secondary base station, respectively.

\section{A. Pathloss Model}

Consider log-distance pathloss model where the received power can be written as

$$
P_{r x}(d)=\frac{P_{t x} \xi 10^{0.1 X}}{d^{\eta}},
$$

where $\eta$ is the pathloss exponent, $d$ is the distance between the transmitter and the receiver, $\xi$ is the pathloss in a reference distance with the transmit and receive antenna gains and the effect of the wavelength. $10^{0.1 X}$ is a log-normal shadowing where $X$ is a Gaussian random variable with zero mean and variance $\sigma_{x}^{2}$. We assume that the measured symbols are averaged out to remove the small-scale fading effect.

Given that log-normal shadowing is present, the interference constraint at the primary user is satisfied in a probabilistic manner. In order to avoid causing a harmful interference at the primary user under log-normal shadowing, we adjust the original interference temperature such that the probability of the interference constraint violation is bounded by a probability of our choice $p_{\epsilon}$. A lower $p_{\epsilon}$ results in a lower adjusted interference temperature, which is more conservative way of protecting the primary user. Thus, we formulate the following:

$$
\operatorname{Pr}\left(10 \log P_{r x}(d)>10 \log I_{t h}\right) \leq p_{\epsilon},
$$

where $\log (\cdot)$ is the $\log$ arithm function with base 10 . Substituting (1) into (2) and using the fact that $\operatorname{Pr}(X>\gamma)=Q(\gamma)$ for Normal distribution, where $Q(\cdot)$ is the $\mathrm{Q}$ function, we get

$$
\frac{P_{t x} \xi}{d^{\eta}}=\frac{I_{t h}}{10^{0.1 Q^{-1}\left(p_{\epsilon} \sigma_{x}\right)}} \text {. }
$$

From (3), we can find the secondary transmitter's maximum transmit power that obeys the interference constraint with a probability $\left(1-p_{\epsilon}\right)$ when log-normal shadowing is present.

\section{B. Downlink}

The distance between the secondary base station and the primary user can be written as

$$
d_{0}=\sqrt{\left(x_{0}^{(c)}-x_{1}^{(p)}\right)^{2}+\left(y_{0}^{(c)}-y_{1}^{(p)}\right)^{2}} .
$$

Given the knowledge of $d_{0}$, the interference power at the primary user based on the pathloss model in (1) is given by

$$
\sum_{k=1}^{K} a_{k, i} b_{i} \frac{p_{k, i} \xi}{d_{0}^{\eta}} \leq \frac{I_{t h}}{10^{0.1 Q^{-1}\left(p_{\epsilon} \sigma_{x}\right)}}, \quad \forall i
$$

where $I_{t h}$ is the maximum interference level tolerable by the primary user and is adjusted based on $p_{\epsilon}, a_{k, i} \in\{0,1\}$ is the subchannel allocation indicator showing that the $i$ th subchannel is allocated to the secondary user $k$. Similarly, $b_{i}$ is the subchannel allocation indicator for the primary user. If $b_{i}=0$, the $i$ th subchannel is not being used by the primary user and therefore the secondary users can use it with free of interference, whereas $b_{i}=1$ indicates that the $i$ th subchannel is being used by the primary user and thus the secondary users may use it under the interference constraint. Let $p_{k, i}$ denote the allocated power by the secondary base station to the secondary user $k$ on the $i$ th subchannel in case of downlink or the transmitted power by the secondary user $k$ on the $i$ th subchannel in case of uplink.

\section{Uplink}

The distance between the secondary user $k$ and the primary user can be computed as

$$
d_{k}=\sqrt{\left(x_{1}^{(p)}-x_{k}^{(c)}\right)^{2}+\left(y_{1}^{(p)}-y_{k}^{(c)}\right)^{2}}, \quad k=1, \cdots, K,
$$

As similar to the downlink case, the interference power at the primary user due to the uplink signal from the secondary user $k$ can be modelled by

$$
\sum_{k=1}^{K} a_{k, i} b_{i} \frac{p_{k, i} \xi}{d_{k}^{\eta}} \leq \frac{I_{t h}}{10^{0.1 Q^{-1}\left(p_{\epsilon} \sigma_{x}\right)}}, \quad \forall i .
$$

The pathloss exponent $\eta$ is assumed to be the same for both downlink and uplink.

\section{Proposed Resource Allocation}

We assume that the orthogonality is maintained between the subchannels used by the secondary users and the subchannels occupied by the primary user's band so that there is no correlation between those subchannels.

\section{A. Downlink}

Our goal is to allocate secondary base station's power and subchannel to secondary users in order to maximize the sum 
rate under the interference constraint at the primary user. The problem under investigation is expressed as

$$
\begin{gathered}
\max \sum_{k=1}^{K} \sum_{i=1}^{L} a_{k, i} \log _{2}\left(1+\frac{\left|h_{k, i}\right|^{2} p_{k, i}}{N_{o}}\right) \\
\text { with subject to } \\
\sum_{k=1}^{K} a_{k, i}=1, \quad \forall i, \\
\sum_{k=1}^{K} \sum_{i=1}^{L} a_{k, i} p_{k, i} \leq P_{t o t}, \\
\sum_{k=1}^{K} a_{k, i} b_{i} \frac{p_{k, i} \xi}{d_{0}^{\eta}} \leq \frac{I_{t h}}{10^{0.1 Q^{-1}\left(p_{\epsilon} \sigma_{x}\right)}}, \quad \forall i,
\end{gathered}
$$

where $d_{0}$ is the distance between the secondary base station and the primary user and $I_{t h}$ is the interference threshold for the primary user. In (9), each subchannel is allocated to only one secondary user. (10) is the total power constraint in the secondary base station and (11) shows the interference constraint at the primary user. Note that secondary users can use the subchannels being occupied by the primary user as long as the interference constraint at the primary user is met.

Using the Lagrange multiplier technique and (8) is formulated as

$$
\begin{aligned}
\mathcal{L} & =\sum_{k=1}^{K} \sum_{i=1}^{L} a_{k, i} \log _{2}\left(1+\frac{\left|h_{k, i}\right|^{2} p_{k, i}}{N_{o}}\right) \\
& +\sum_{i=1}^{L} \lambda_{i}\left(\frac{I_{t h}}{10^{0.1 Q^{-1}\left(p_{\epsilon} \sigma_{x}\right)}}-\sum_{k=1}^{K} a_{k, i} b_{i} \frac{p_{k, i} \xi}{d_{0}^{\eta}}\right) \\
& +\rho_{0}\left(P_{\text {tot }}-\sum_{k=1}^{K} \sum_{i=1}^{L} a_{k, i} p_{k, i}\right),
\end{aligned}
$$

where $\lambda_{i}$ and $\rho_{0}$ are positive Lagrangian coefficients. The Karush-Kuhn-Tucker (KKT) conditions [11] are listed as:

$$
\begin{aligned}
\frac{a_{k, i}\left|h_{k, i}\right|^{2}}{N_{o}+\left|h_{k, i}\right|^{2} p_{k, i}}-a_{k, i} b_{i} \frac{\lambda_{i} \xi}{d_{0}^{\eta}}-a_{k, i} \rho_{0} & =0, \\
\lambda_{i}\left(\frac{I_{t h}}{10^{0.1 Q^{-1}\left(p_{\epsilon} \sigma_{x}\right)}}-\sum_{k=1}^{K} a_{k, i} b_{i} \frac{p_{k, i} \xi}{d_{0}^{\eta}}\right) & =0, \\
\rho_{0}\left(P_{\text {tot }}-\sum_{k=1}^{K} \sum_{i=1}^{L} a_{k, i} p_{k, i}\right) & =0 .
\end{aligned}
$$

Even though the above problem is in the form of mixed-integer programming problem, which is in general NP-hard, a simple 2 step approach in fact provides the maximum capacity thanks to the total power constraint. Since the goal is to maximize the capacity, the first step is to allocate each subchannel into the secondary user with the maximum SNR for that subchannel. Thus, the subchannel allocation indicator for secondary users is simply given as $a_{k, i}=1$ for $k=k_{i}$, or 0 otherwise, where

$$
k_{i}=\underset{k}{\arg \max } \frac{\left|h_{k, i}\right|^{2}}{N_{o}}, \quad \forall i .
$$

Let $\mathcal{U}$ denote the set of unallocated subchannels and $\mathcal{U}_{p}$ the set of the subchannels occupied by the primary user. It is assumed that $\mathcal{U}_{p}$ is already known by spectrum sensing. Thus, $\mathcal{U}_{c}=$ $\mathcal{U}-\mathcal{U}_{p}$ is the set of the interference-free subchannels. The subchannel allocation indicator for the primary user is given as $b_{i}=1$ for $i \in \mathcal{U}_{p}$ or 0 for $i \in \mathcal{U}_{c}$.

In the second step, substituting $a_{k, i}$ and $b_{i}$ into (13), the optimal transmit power can be obtained as

$$
p_{k_{i}, i}=\left[\frac{d_{0}^{\eta}}{\lambda_{i} \xi+\rho_{0} d_{0}^{\eta}}-\frac{N_{o}}{\left|h_{k_{i}, i}\right|^{2}}\right]^{+}, \quad \forall i .
$$

where $[x]^{+}=\max \{x, 0\}$. From (14), it is clear that $\lambda_{i}=0$ for $i \in \mathcal{U}_{c}$. Therefore, (17) can be simplified as

$$
p_{k_{i}, i}= \begin{cases}{\left[\frac{1}{\rho_{0}}-\frac{N_{o}}{\left|h_{k_{i}, i}\right|^{2}}\right]^{+},} & i \in \mathcal{U}_{c}, \\ \min \left\{\left[\frac{1}{\rho_{0}}-\frac{N_{o}}{\left|h_{k_{i}, i}\right|^{2}}\right]^{+}, \frac{I_{t h}^{\prime} d_{0}^{\eta}}{\xi}\right\}, & i \in \mathcal{U}_{p},\end{cases}
$$

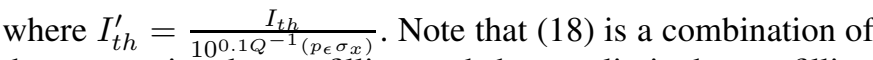
the conventional waterfilling and the cap-limited waterfilling both with the common water level shown as follows:

$$
\frac{1}{\rho_{0}}=\frac{1}{|\mathcal{U}|}\left(P_{t o t}-\left|\mathcal{S}_{p}\right| \frac{I_{t h}^{\prime} d_{0}^{\eta}}{\xi}+\sum_{i \in \mathcal{U}} \frac{N_{0}}{\left|h_{k_{i}, i}\right|^{2}}\right),
$$

where $|\mathcal{U}|$ denotes the size (number of elements) of the set $\mathcal{U}$ and $\mathcal{S}_{p}$ is the set of the subchannels satisfying the following conditions: $\frac{1}{\rho_{0}}-\frac{N_{0}}{\left.h_{k_{i}, i}\right|^{2}}>\frac{I_{t h}^{\prime} d_{0}^{\eta}}{\xi}$.

Using the sets $\mathcal{U}, \mathcal{U}_{p}$, and $\mathcal{S}_{p}$ shown earlier, the power allocation algorithm runs as follows. The sets are initialized as $\mathcal{U}=\{1, \cdots, L\}$ and $\mathcal{S}_{p}=\{\}$. First, perform the conventional water filling algorithm and compute (19) and $p_{k, i}=\frac{1}{\rho_{0}}-$ $\frac{N_{o}}{\left|h_{k_{i}, i}\right|^{2}}$. If $p_{k, i}<0, \exists i \in \mathcal{U}$, then $\mathcal{U}=\mathcal{U}-\{i\}$ and redo the above calculations. If $\frac{p_{k, i} \xi}{d_{0}^{\eta}}>I_{t h}^{\prime}$ for any $i \in \mathcal{U}_{p}$, the allocated power will be saturated such that $p_{k, i}=\frac{I_{t h}^{\prime} d_{0}^{\eta}}{\xi}$. Then, update $\mathcal{U}=\mathcal{U}-\{i\}, \mathcal{U}_{p}=\mathcal{U}_{p}-\{i\}$, and $\mathcal{S}_{p}=\mathcal{S}_{p}+\{i\}$. Recalculate $\frac{1}{\rho_{0}}$ (increasing water level) using (19). Repeat the above procedure until no subchannel $i \in \mathcal{U}_{p}$ that satisfies $\frac{p_{k, i} \xi}{d_{0}^{\prime \prime}}>I_{t h}^{\prime}$ is found.

\section{B. Uplink}

Similarly, our goal is to maximize the sum rate under the individual secondary user power constraint. The problem is formulated as

$$
\begin{gathered}
\max \sum_{k=1}^{K} \sum_{i=1}^{L} a_{k, i} \log _{2}\left(1+\frac{\left|h_{k, i}\right|^{2} p_{k, i}}{N_{o}}\right) \\
\text { with subject to } \\
\sum_{k=1}^{K} a_{k, i}=1, \quad \forall i \\
\sum_{i=1}^{L} a_{k, i} p_{k, i} \leq P_{k}, \quad \forall k, \\
\sum_{k=1}^{K} a_{k, i} b_{i} \frac{p_{k, i} \xi}{d_{k}^{\eta n}} \leq \frac{I_{t h}}{10^{0.1 Q^{-1}\left(p_{\epsilon} \sigma_{x}\right)}}, \quad \forall i
\end{gathered}
$$

where $P_{k}$ is the transmit power budget for the secondary user $k$, and $d_{k}$ is the distance between the primary user and the secondary user $k$. (22) is the individual power constraint for the secondary user $k$.

Unlike the downlink case, it is not optimal in the uplink case to separate subchannel and power allocations due to the per-user power constraint. In order to solve the problem with a reduced complexity, we propose an algorithm that runs a 
per-subchannel 2 step procedure of user selection and power allocation for all the subchannels one after another. A brief description of the procedure is given as follows.

1) Initialize $\mathcal{U}=\{1, \cdots, L\}, \mathcal{U}_{k}=\{1, \cdots, L\}$ and $\mathcal{S}_{k}=$ \{\}$, \forall k$, where $\mathcal{U}_{k}$ and $\mathcal{S}_{k}$ are the set of the unallocated subchannels for the user $k$ and the set of the subchannels that their allocated power should be capped due to interference constraint, respectively.

2) Run the cap-limited waterfilling algorithm over the available subchannels for each user independently. The water level for secondary user $k$ is shown as

$$
\frac{1}{\rho_{k}}=\frac{1}{\left|\mathcal{U}_{k}\right|}\left(P_{k}-\left|\mathcal{S}_{k}\right| \frac{I_{t h}^{\prime} d_{k}^{\eta}}{\xi}+\sum_{i \in \mathcal{U}_{k}} \frac{N_{0}}{\left|h_{k, i}\right|^{2}}\right) \text {. }
$$

For each user $k$, if $i \in \mathcal{U}_{p}$ and $p_{k, i}>\frac{I_{t h}^{\prime} d_{k}^{\eta}}{\xi}$, then $p_{k, i}=$ $\frac{I_{t h}^{\prime} d_{k}^{\eta}}{\xi}$ and $\mathcal{S}_{k}=\mathcal{S}_{k}+\{i\}$.

3) Compute the capacity for each subchannel and user as

$$
C_{k, i}=\log _{2}\left(1+\frac{p_{k, i}\left|h_{k, i}\right|^{2}}{N_{o}}\right),
$$

and then select the pair with the highest capacity as $\left\{k_{i}^{*}, i^{*}\right\}=\arg \max _{k, i \in \mathcal{U}} C_{k, i}$.

4) Allocate the $i^{*}$ th subchannel to the user $k_{i}^{*}\left(a_{k_{i}^{*}, i^{*}}=1\right)$.

5) Remove this subchannel from the sets $\mathcal{U}=\mathcal{U}-\left\{i^{*}\right\}$ and $\mathcal{U}_{k}=\mathcal{U}_{k}-\left\{i^{*}\right\}$, where $k=1, \cdots, i^{*}-1, i^{*}+1, \cdots, K$.

6) Repeat the above procedure until $\mathcal{U}$ is empty.

Notice that, unlike the downlink case, the user with the maximum SNR for a subchannel can not always offer the highest rate in the uplink case. Therefore, to maximize the capacity, each subchannel needs to be allocated into the secondary user with the highest capacity for the subchannel.

Using the Lagrangian and KKT conditions, the optimal transmit power can be obtained as

$$
p_{k_{i}, i}=\left[\frac{d_{k_{i}}^{\eta}}{\lambda_{i} \xi+\rho_{k_{i}} d_{k_{i}}^{\eta}}-\frac{N_{o}}{\left|h_{k_{i}, i}\right|^{2}}\right]^{+}, \quad \forall i
$$

which can be simplified as

$$
p_{k_{i}, i}= \begin{cases}{\left[\frac{1}{\rho_{k_{i}}}-\frac{N_{o}}{\left|h_{k_{i}, i}\right|^{2}}\right]^{+},} & i \in \mathcal{U}_{c}, \\ \min \left\{\left[\frac{1}{\rho_{k_{i}}}-\frac{N_{o}}{\left|h_{k_{i}, i}\right|^{2}}\right]^{+}, \frac{I_{t h}^{\prime} d_{k_{i}}^{\eta}}{\xi}\right\}, & i \in \mathcal{U}_{p} .\end{cases}
$$

\section{Presence of Correlation Between Subchannels}

Due to the absence of frequency orthogonality, the signal power of the secondary users on the subchannels may show a correlation to the primary user's band in the form of inter-carrier interference (ICI). Since the spectrum of each subcarrier in OFDM demonstrates a sinc function, the envelope of the side lobe peaks follows an exponential decay. Thus, we model the ICI coefficient between the $i$ th and the $j$ th subchannels as $g_{i, j}=\frac{\alpha}{(i-j)^{2}}$, where $g_{i, i}=1, \forall i$, and $\alpha$ is a system-dependent constant determined based on the size of subchannel.
The problems for downlink and uplink with ICI are formulated by replacing (11) and (23) with

$$
\sum_{k=1}^{K} \frac{b_{i} \xi}{d_{0}^{\eta}} \sum_{j=1}^{L} g_{i, j} a_{k, j} p_{k, j} \leq \frac{I_{t h}}{10^{0.1 Q^{-1}\left(p_{\epsilon} \sigma_{x}\right)}}, \quad \forall i
$$

and

$$
\sum_{k=1}^{K} \frac{b_{i} \xi}{d_{k}^{\eta}} \sum_{j=1}^{L} g_{i, j} a_{k, j} p_{k, j} \leq \frac{I_{t h}}{10^{0.1 Q^{-1}\left(p_{\epsilon} \sigma_{x}\right)}},
$$

respectively. Similar to the earlier cases, the Lagrangian and and KKT conditions allow the optimal transmit power to be obtained for downlink and uplink as

$$
p_{k_{i}, i}=\left[\frac{d_{0}^{\eta}}{\xi \sum_{j \in \mathcal{U}_{p}} g_{j, i} \lambda_{j}+\rho_{0} d_{0}^{\eta}}-\frac{N_{o}}{\left|h_{k_{i}, i}\right|^{2}}\right]^{+},
$$

and

$$
p_{k_{i}, i}=\left[\frac{d_{k_{i}}^{\eta}}{\xi \sum_{j \in \mathcal{U}_{p}} g_{j, i} \lambda_{j}+\rho_{k_{i}} d_{k_{i}}^{\eta}}-\frac{N_{o}}{\left|h_{k_{i}, i}\right|^{2}}\right]^{+},
$$

respectively. These equations indicate that each subchannel uses a less power due to the correlation, which is intuitively understood.

Substituting (30) into (10) and (28), we get $\left(\left|\mathcal{U}_{p}\right|+1\right)$ equations as

$$
\sum_{k=1}^{K} \sum_{i=1}^{L} \frac{a_{k, i} d_{0}^{\eta}}{\xi \sum_{j \in \mathcal{U}_{p}} g_{j, i} \lambda_{j}+\rho_{0} d_{0}^{\eta}}-\frac{a_{k, i} N_{o}}{\left|h_{k, i}\right|^{2}}=P_{t o t},
$$

and

$$
\sum_{k=1}^{K} \sum_{j=1}^{L} \frac{g_{i, j} a_{k, j} d_{0}^{\eta}}{\xi \sum_{n \in \mathcal{U}_{p}} g_{n, j} \lambda_{n}+\rho_{0} d_{0}^{\eta}}-\frac{g_{i, j} a_{k, j} N_{o}}{\left|h_{k, j}\right|^{2}}=\frac{I_{t h}^{\prime} d_{0}^{\eta}}{\xi}, i \in \mathcal{U}_{p},
$$

from which $\lambda_{1}, \cdots, \lambda_{\left|\mathcal{U}_{p}\right|}$ and $\rho_{0}$ are obtained and used to compute $p_{k_{i}, i}$ for downlink using (30). Similarly, substituting (31) into (22) and (29) delivers $\left(\left|\mathcal{U}_{p}\right|+K\right)$ equations as

$$
\sum_{i=1}^{L} \frac{a_{k, i} d_{k}^{\eta}}{\xi \sum_{j \in \mathcal{U}_{p}} g_{j, i} \lambda_{j}+\rho_{k} d_{k}^{\eta}}-\frac{a_{k, i} N_{o}}{\left|h_{k, i}\right|^{2}}=P_{k}, \quad k=1, \cdots, K
$$

and

$\sum_{k=1}^{K} \sum_{j=1}^{L} \frac{g_{i, j} a_{k, j} d_{k}^{\eta}}{\xi \sum_{n \in \mathcal{U}_{p}} g_{n, j} \lambda_{n}+\rho_{k} d_{k}^{\eta}}-\frac{g_{i, j} a_{k, j} N_{o}}{\left|h_{k, j}\right|^{2}}=\frac{I_{t h}^{\prime} d_{k}^{\eta}}{\xi}, i \in \mathcal{U}_{p}$,

from which we can compute $\lambda_{1}, \cdots, \lambda_{\left|\mathcal{U}_{p}\right|}$ and $\rho_{1}, \cdots, \rho_{K}$. $p_{k, i}$ for uplink are obtained by substituting these into (31).

\section{Numerical and Simulation Results}

Extensive simulations are performed, where the cognitive radio system is assumed to have total 64 subchannels. Base station transmit power budget of $20 \mathrm{dBm}$, user transmit power budget of $3 \mathrm{dBm}, I_{t h}=-100 \mathrm{dBm}$, the noise floor of $-110 \mathrm{dBm}$, and pathloss model $P L[\mathrm{~dB}]=100+30 \log _{10}\left(d_{k m}\right)+X$ are assumed, where $X$ is log-normal shadowing.

We consider two different simulation scenarios for the secondary users and the primary user: 1) All the secondary users are located along the circle with a radius of $1(\mathrm{~km})$ and the primary user is located at random within the cell with the radius of $8 \mathrm{~km}$, and 2) The secondary users as well as the 


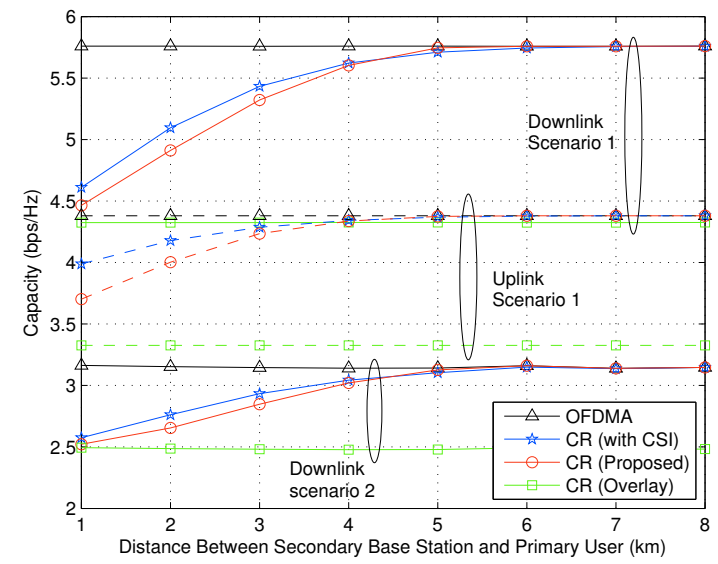

Fig. 1. Capacity of various schemes as a function of distance between the secondary base station and the primary user when $p_{\epsilon}=0.023$.

primary user are randomly distributed within the cell. This is a more practical scenario with non-identically distributed users in a cellular environment.

Fig. 1 compares the capacity of various resource allocation algorithms as a function of the distance between the secondary base station and the primary user. It is assumed that 20 secondary users are present in the cell and the primary user's band spans over 20 subchannels. For each scenario, the proposed location-based resource allocation algorithm indeed shows a capacity which is close to that of the ideal algorithm where CSI of the interference link is available. The capacity of uplink scenario 2 is not plotted because all the systems considered show the capacity of around $1(\mathrm{bps} / \mathrm{Hz})$ with no much difference, which is understood by the fact that more number of users are located close to the cell boundary in a cellular environment. We have also performed exhaustive search algorithms which show that the proposed downlink algorithm indeed provides the optimal capacity and the uplink algorithm achieves a near-optimal capacity. Due to space limitation, the figure for the optimality is omitted.

Fig. 2 shows the impact of $p_{\epsilon}$ on the performance of the location-based algorithm. As in the figure, the lower $p_{\epsilon}$, the stronger protection we put in place for the primary user and therefore the secondary users more tends to avoid the subchannels under the primary user's band. Fig. 3 shows the impact of correlation between subchannels on the capacity of the proposed resource allocation algorithm.

\section{CONCLUSION}

This paper introduces a resource allocation algorithm based on location information for OFDMA cognitive radio systems and shows that it achieves a near-optimal capacity even without any CSI of the interference link. Since the interference constraint is met in a probablistic sense in the presence of log-normal shadowing, the proposed algorithm is formulated to control the probability of interference constraint violation. We also consider a correlation between subchannels in the formulation, which occurs due to the absence of spectral orthogonality between the primary and the secondary users

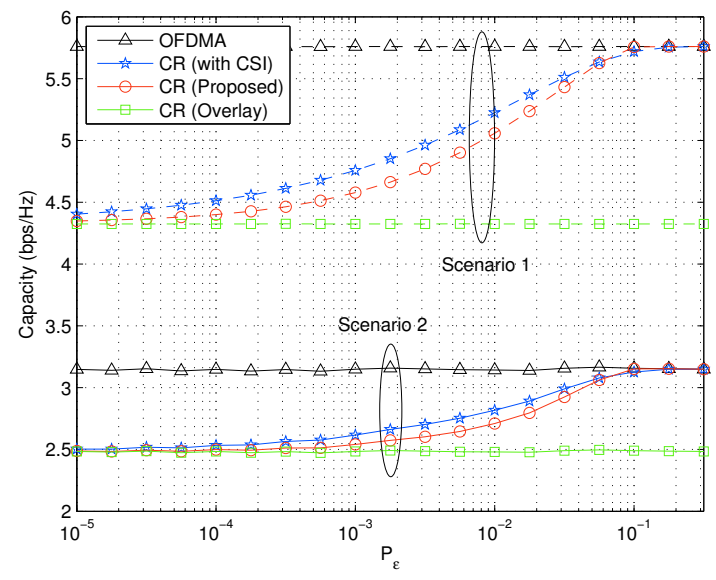

Fig. 2. Downlink capacity of various schemes as a function of $p_{\epsilon}$ when the distance between the primary user and the secondary base station is $3(\mathrm{~km})$.

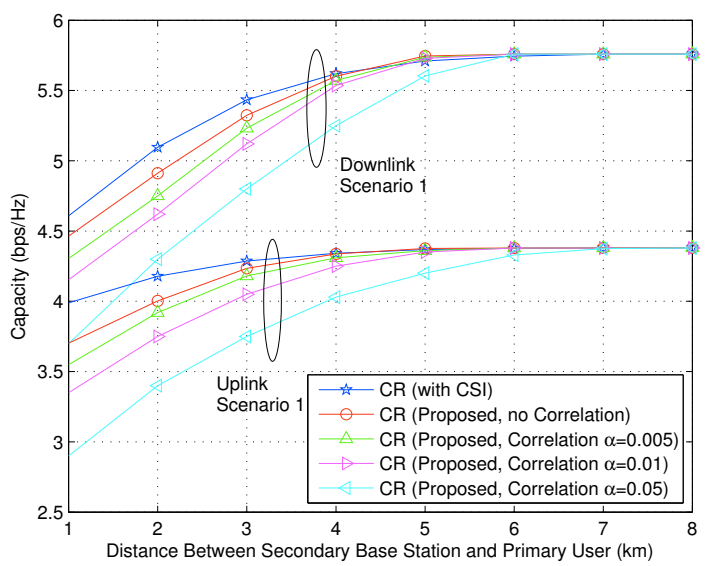

Fig. 3. Impact of correlation between subchannels on the capacity.

and erodes the performance significantly.

\section{REFERENCES}

[1] J. Mitola and G. Q. Macguire Jr., "Cognitive radio: Making software radios more personal," IEEE Pers. Commun. Mag., vol. 6, no. 4, pp. 13-18, Aug. 1999.

[2] S. Haykin, "Cognitive radio: brain-empowered wireless communications," IEEE J. Select. Areas Commun., vol. 23, no. 2, pp. 201-220, Feb. 2005.

[3] H. Celebi and H. Arslan, "Utilization of Location Information in Cognitive Wireless Networks," IEEE Wireless Commun. Mag., vol. 14, no. 4, pp. 6-13, Aug. 2007.

[4] O. Duval, A. Punchihewa, F. Gagnon, C. Despins, and V. K. Bhargava, "Blind multi-sources detection and localization for cognitive radios," Proc. IEEE Globe Commun. Conf., vol. 27, Nov. 2008, pp. 2962-2966.

[5] S. Kim, H. Jeon, and J. Ma, "Robust localization with unknown transmission power for cognitive radio," in Proc. IEEE MILCOM, Oct. 2007.

[6] H. Celebi and H. Arslan, "Cognitive Positioning Systems," IEEE Trans. Wireless Commun., vol. 6, no. 12, pp. 4475-4483, Dec. 2007.

[7] F. F. Digham, "Joint power and channel allocation for cognitive radios," Proc. IEEE Wireless Commun. Networking Conf., 2008, pp. 882-887.

[8] P. Cheng, Z. Zhang, H.-H. Chen, and P. Qiu, "Optimal distributed joint frequency, rate and power allocation in cognitive OFDMA systems," IET Commun., vol. 2, no. 6, pp. 815-826, 2008.

[9] M. Shaat, and F. Bader, "A Two-Step Resource Allocation Algorithm in Multicarrier Based Cognitive Radio System," in Proc. IEEE Wireless Commun. Networking Conf., Apr. 2010, pp. 18-21.

[10] Federal Communications Commission: "Spectrum policy task force," Report ET Docket, Nov. 2002.

[11] S. Boyd and L. Vandenberghe, Convex Optimization, Cambridge University Press, 2004. 